\title{
Dentistry, the press and a pandemic
}

\author{
Matthew J. Sedgwick ${ }^{\star 1}$ and Deborah Krishnapillai ${ }^{1}$
}

\section{Key points}

Newspapers are able to sway public opinion and can also influence health professionals on major issues.
Open and honest discourse existed, with many dentists and dental organisations liaising with newspaper journalists about health issues facing patients and the dental profession.
The relationship between the press and the dental profession is dynamic and fluid, with some articles sensationalising misconduct and others being informative, balanced and helpful.

\begin{abstract}
Introduction Newspapers are a valuable source of information for many people and report on a variety of topics including dentistry. During the COVID-19 pandemic, where there has been a considerable amount of information seeking from health professionals and the public alike, this study examines how dentistry has been represented in the media.

Methods and materials Articles which involve dentistry were searched for between 23 March 2020 and 31 December 2020 across multiple newspaper websites. Qualitative analysis was carried out of the data obtained to identify themes.

Results In total, 179 articles were included in the study and themes identified. These related to access to dental services, do-it-yourself (DIY) dentistry, dental pain and emergency care, financial concerns and practice closures, oral health, personal protective equipment, as well as the return to work in June. There were very few stories about dental misconduct.
\end{abstract}

Conclusions Constructive collaboration between newspapers and dentists existed as many articles were promoting positive oral health messages, discussed problems facing dentists re-opening as well as issues many patients faced during the first lockdown period.

\section{Introduction}

A recently published article discusses how dentists' behaviour is portrayed in newspapers in the UK and found that it is often sensationalised and focused on immoral or dishonest behaviour. ${ }^{1}$ There has always been a view from those cynical of the press that the stories are designed to have 'click-bait' titles and stories to entice the readers. Unfortunately, even with this cynicism, people can be swayed and form opinions based on the stories published.

'Department of Oral and Maxillofacial Surgery, John Radcliffe Hospital, Headley Way, Headington, Oxford, Oxfordshire, OX3 9DU, UK

*Correspondence to: Matthew Sedgwick

Email address: matthew.sedgwick@nhs.net

Refereed Paper.

Accepted 5 May 2021

https://doi.org/10.1038/s41415-021-3734-0
Dentistry is still struggling to shake off the image of an uncaring, sadistic profession who enjoy 'pulling teeth with a knee on the patient's chest' as commonly described by many an anxious patient. ${ }^{2}$ As dentists, we are aware that doing so still would not gain sufficient leverage for a stubborn tooth, but as it is repeated and spread among patients, this sort of story becomes embedded in the subconscious mind. Comedians, film makers and television shows have often portrayed dentists in this negative way and it has been difficult to alter this perception ever since.

Unifying healthcare messages across competing newspapers would be extremely challenging due to differences in agendas for journalists and health care professionals. It is important to try to work with the media and challenge them if they publish inaccurate or misleading stories. Journalists in one study were interviewed and they commented that their aims were to be informative and critical but reporting stories promptly was a priority over the social impact it may have (apart from in exceptional circumstances) and acknowledged headlines were often designed to be dramatic. ${ }^{3}$ It is important for healthcare professionals to understand the potential implications of what they say and be aware of how they conduct themselves when working with the media. If messages are confusing, misleading, or contradictory then the desired impact may even be the opposite to what was hoped for.

During the COVID-19 pandemic, informationseeking behaviour has increased dramatically and interest in health care news stories has grown. This has led to a barrage of media coverage, constantly updating the public with the latest developments and recommendations. With people furloughed or isolating, there is the potential to read a large volume of news stories as they spend more time on their electronic devices. However, there is a saturation point, as many people can end up feeling depressed and demoralised with the glut of 
COVID-19 stories. Research from Pew Research Centre found that about $71 \%$ of Americans say they need breaks from COVID-19 stories. ${ }^{4}$ This means that stories regarding other issues may gain more attention from casual readers. This is an audience that dentists could reach, with important messages to help the audience understand what to do about their oral health at a time where access was difficult for many. Also, it could be used to increase understanding of why dentistry was changing in many ways and why a routine examination was no longer routine (or even available at all!)

The objective of the study is to identify published articles relating to UK dentistry during the ongoing COVID-19 pandemic and analyse the content thematically.

\section{Methods and materials}

This is a cross-sectional study of articles spanning from 23 March 2020 when the first national lockdown was introduced up until 31 December 2020. The following newspapers were selected as they are the most circulated in UK: ${ }^{5}$

- The Guardian

- The Times

- The Daily Mail/Mail online

- The Sun

- The Mirror

- Metro.

Articles were screened for relevance and were included if they were associated with UK Dentistry within the specified time range. Data were collected sequentially and entered onto a Microsoft Excel spreadsheet via codes and subsequently, themes were identified. Codes and themes were identified by both reviewers and an agreement reached.

Articles were excluded if they were not related to dentistry in the UK or were duplicated in the same newspaper.

Table 1 Number of articles discussing dentistry in the UK

\begin{tabular}{l|l}
\hline Name of newspaper & Number of articles \\
\hline The Guardian & 11 \\
\hline The Times & 24 \\
\hline The Daily Mail & 65 \\
\hline The Sun & 48 \\
\hline The Mirror & 12 \\
\hline Metro & 19
\end{tabular}

\section{Results}

A total of 179 articles were identified across the six selected newspapers (Table 1). The Daily Mail/Mail online was the most active, publishing 65 dental-related articles. The least active was The Guardian with 11 articles. Multiple themes existed in the articles and there was a total of 606 code entries for the articles (Tables 2 and 3 ).

The following themes were identified:

- Access

- Do-it-yourself (DIY) dentistry

- Dental pain

- Financial

- Oral health

- Personal protective equipment (PPE)

- Return to work.

\section{Access}

Access to dentists was the most common theme across all newspapers and articles. There were two subthemes associated with this theme; appointment restrictions and treatment delays.

Appointment restrictions were discussed when practices were told to start re-opening, as the British Dental Association (BDA) and individual dentists communicated across media platforms that patients were not to expect a full service and routine appointments for some time: 'the BDA said that there was no possibility of the service carrying out anything more than a fraction of the 39.72 million courses of treatment that were provided by NHS dentists in England in 2018-2019.'

Treatment delays were discussed alongside the appointment restrictions that were in place as practices sourced personal protective equipment (PPE) and waited on guidance from regulatory bodies: 'patients are facing a twoyear delay for dental surgery as waiting times have doubled in the last year because of the COVID-19 pandemic.'

Table 2 Number of coded entries for main themes identified

\begin{tabular}{l|l} 
Theme & Number of content codes \\
\hline Dental pain & 67 \\
\hline Access & 56 \\
\hline Financial & 47 \\
\hline Oral health & 43 \\
\hline DIY dentistry & 39 \\
\hline PPE & 36 \\
\hline Return to work & 29 \\
\hline
\end{tabular}

\section{Do-it-yourself dentistry}

These articles were related to patients having to perform dental treatment on their own teeth. This ranged from applying a temporary filling to extractions. Often these stories would have an interview with patients who were unable to access dental care due to closing of the practices during the first few months of the pandemic. Clickbait headlines often included details of the individual's family circumstances and would use emotive language such as 'dad downed eight Stellas and pulled his own teeth out as he couldn't see dentist'. Another article discussed parental worries and stated 'fears of spikes in DIY dentistry as parents ask how to remove their kid's teeth.' One article did offer some useful advice on popular home remedies for common sources of dental and facial pain, along with warnings of when these were not appropriate and when it would be best to seek professional intervention. ${ }^{10}$

\section{Dental pain}

A total of 67 articles had themes related to dental pain or issues. Of these, 37 articles were related to toothache suffered by a patient, 19 related to emergency care requirements, 8 discussed dental caries or poor-quality teeth and 3 discussed severe infections; however, this was related to the same patient, where 'a single mother narrowly escaped death and spent five months in hospital after an abscess on her tooth led to a life-threatening infection in her brain." ${ }^{11}$

\section{Financial}

Articles often discussed the impact that the pandemic has had on being able to pay staff and concerns about being able to afford to stay open with rising costs of PPE. For example, 'in a shocking statement, the BDA, which represents many of the UK's 12,000 dental practices, has warned that up to one in five of them could close permanently in the next few weeks because of the repercussions of the COVID-19 pandemic.' ${ }^{\prime 2}$ There were eight articles related to potential practice closures.

Table 3 Number of coded entries for subthemes

\begin{tabular}{l|l} 
Subtheme & $\begin{array}{l}\text { Number of } \\
\text { content codes }\end{array}$ \\
\hline Appointment restrictions & 34 \\
\hline Fear/concern for the future & 22 \\
\hline Treatment delays & 21 \\
\hline Lack of government help/guidance & 12 \\
\hline Lack of preparation & 7 \\
\hline
\end{tabular}




\section{Oral health}

Oral health was a recurring theme, especially early on in the pandemic, where dental professionals would give oral hygiene advice or home care advice for patients who could not access their dentist due to practice closures. In total, 12 articles included detailed hygiene instructions and 6 covered toothbrushing. One article did address the possibility of mask mouth suggesting the theory that consistent maskwearing could cause a build-up of bacteria or dry mouth, which in turn could lead to issues such as bad breath, decaying teeth or receding gum lines. ${ }^{13}$ It also offered sound advice of maintaining optimal oral hygiene, staying hydrated and smoking cessation as well.

\section{Personal protective equipment}

Articles were predominantly related to a lack of PPE, which was a recurring theme when practices were told to re-open, as many struggled to obtain PPE during a shortage. In total, 16 articles discussed PPE in general terms, such as how it will now be used during patient appointments and patient charges for use of PPE while they visit the practice (patient charges were for private practices). There were a range of charges for PPE, from $£ 25$ to $£ 60 .{ }^{14,15,16}$

\section{Return to work}

This was a major theme and articles were mainly published just before 8 June 2020 when practices were opening. There were three subthemes: 1) fear/concern for the future; 2) lack of preparation; and 3) a lack of government help/guidance. Lack of government help was discussed in 12 articles where dentists felt they were not being helped with PPE costs and seven articles discussed feeling underprepared as a result. Fear/concern for the future was mentioned 22 times. One dentist discusses that 'while we wanted to open and a lot of practices have been gearing themselves up for this, we didn't really have the guidance to tell us what we needed to alter and how we needed to do things. ${ }^{\text {' }}$

\section{Discussion}

The study found that the quality of information being reported was generally helpful and positive towards the dental profession. This is in line with comments made by the Leask study mentioned earlier. ${ }^{3}$ Dental professionals and organisations, especially the British Dental Association, were very actively involved in discussions with journalists and oral health advice was discussed frequently, as well as the difficulties the profession was facing. It was encouraging to see such a productive relationship with the media during a time where it was in the best interests of patients and dentists to provide accurate and helpful information.

The most common themes can be linked together in multiple articles as they go hand in hand with the issues being faced by patients and dentists. Without access to dental practices, the rise of DIY dentistry and concerns with regards to toothache and emergency care were commonly discussed. The articles highlight that there were issues before the coronavirus pandemic accessing NHS dentistry and the increasing backlog of treatments would only worsen the situation. This would be exacerbated by people missing their regular check-ups due to practices being closed, or only treating emergency cases and then suffering from worsening dental diseases.
As the pandemic developed and dental practices re-opened, there was a shift towards returning to work including sourcing PPE, a lack of help from the government and financial concerns. There were concerns with being able to afford to remain open due to a reduced patient case load; however, there were no stories of actual practices being forced to close within this study selection. As expected, PPE was a hot topic due to rising costs and a lack of availability. Early stories discussed the difficulties in acquiring the quantity required to be able to see the volume of patients needing treatment. Further on, towards the end of the year, the stories around PPE seemed to decline as supplies had increased and costs started to decrease. The cycle of themes followed the early stages of lockdown, with dental practices opening again, as well as when the tiered system was introduced (Fig. 1) and this is also reflected in the number of articles published (Fig. 2).

\section{Fig. 1 Distribution of main themes by month}

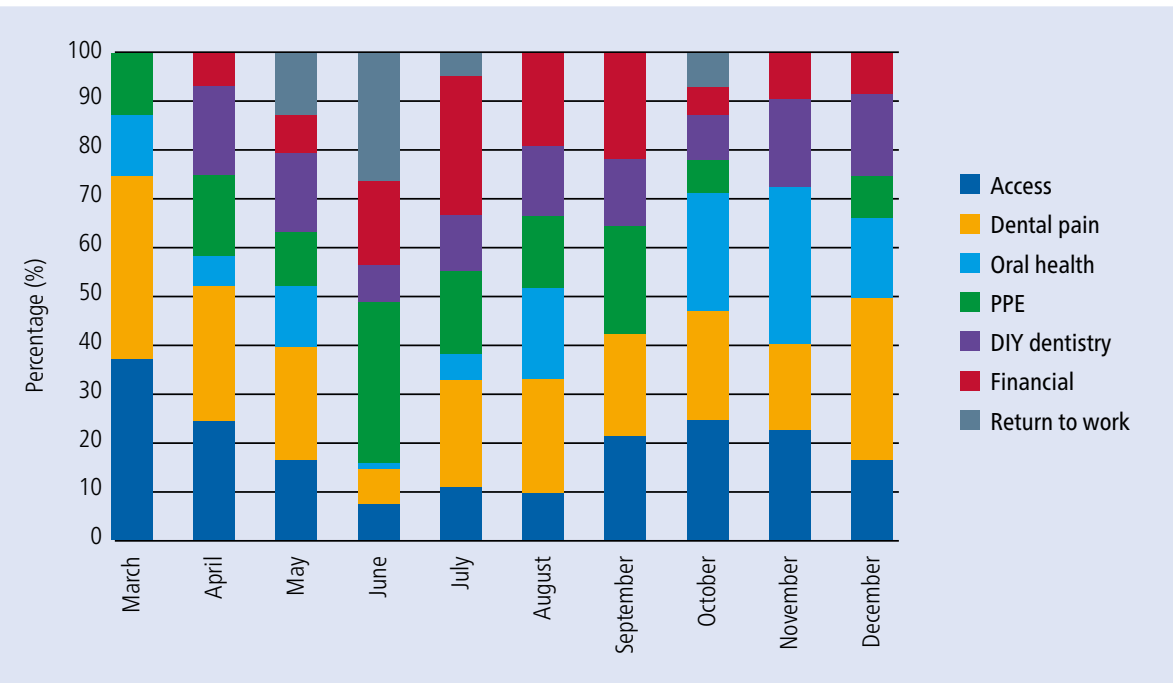

Fig. 2 Number of articles published by month

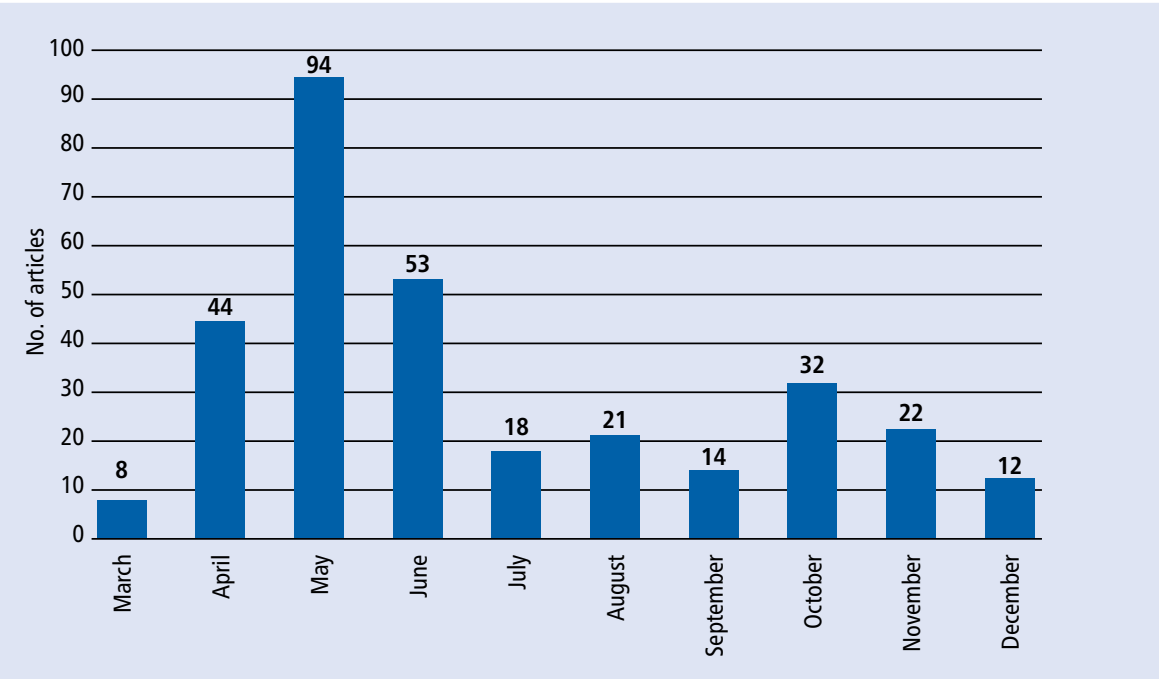


Other studies have found that the media sensationalise stories and health care issues; however, these articles seemed to be in a minority in this study and the balance of information provided throughout the pandemic was fair and generally helpful. ${ }^{18,19}$ These articles tended to be related to DIY dentistry and toothache, as highlighted in the examples of a man requiring a substantial amount of alcohol before removing his own tooth.

Sampling six national newspapers could be seen as a limitation, as there may have been a difference between the data themes generated compared to local or regional ones. However, these are six of the most circulated and popular newspapers in the UK and include both tabloids and broadsheets, so could be considered a representative sample of national interests. Increasing the number of newspapers analysed could have improved thematic saturation and reduced the risk of selection bias, but this is not guaranteed. A large number of the content codes generated were unrelated to each other or the main themes due to the diverse range of topics covered in each article. The lack of advanced search filters on the websites, requiring manual screening of a lot of irrelevant articles, resulted in a technical limitation.

The study shows that the dental profession can help guide public perception of oral health issues via constructive collaboration with newspapers and media sources. Fostering good relationships and being open in communication can improve the quality of information that is available. Guidance is available for dental professionals if speaking to newspapers and it is helpful to prepare beforehand. The journalist should be asked what the content of the discussion will be and you should remain professional, adhere to the General Dental Council's standards and remember to avoid saying anything that could be misquoted or interpreted by someone else in a different way. ${ }^{20,21}$

If statements are given that conflict with other dental professionals or organisations, this may undermine the confidence of the public. Keep them simple and in lay terms to help get important messages across and consider signposting a journalist to clinical experts or professional bodies who may be able to help with their article, such as the British Dental Association, British Dental Health Foundation, a key opinion leader or a specialist in that field. ${ }^{22}$ Specialist societies have press releases about certain topics and are a good source of information for journalists.

\section{Conclusion}

Newspapers often worked with dentists to ensure an accurate reflection of the state of UK dentistry was presented to the general population. Access to dentists, DIY dentistry and tooth-related issues were the main themes, as expected with practice closures. Dentists should continue to be actively involved with newspapers to help improve public image and discuss professional issues in a responsible manner. Further studies could compare the themes during the COVID-19 pandemic to newspaper articles pre-pandemic to see if there is a difference in the type of reporting and number of published articles. There were still some stories about dental misconduct or cosmetic treatments for celebrities; however, these were a small minority.

\section{Ethics declaration}

The authors confirm that there are no conflicts of interests.

\section{Author contributions}

Matthew Sedgwick: conceptualisation, methodology, investigation, writing original draft and formal analysis. Deborah Krishnapillai: conceptualisation, investigation, writing - review and editing and formal analysis.

\section{References}

1. Ellis M, Johnson I. Exploring dentists' professional behaviours reported in United Kingdom newspaper media. Br Dent J 2020; DOI: 10.1038/ s41415-020-1987-7.

2. Payet C. Dental myth: knee on the chest to pull a tooth? 2013. Available at https://www.smilesbypayet. com/2013/02/dental-myth-knee-chest-pull-teeth/ (accessed January 2021).
3. Leask J, Hooker C, King C. Media coverage of health issues and how to work more effectively with journalists: a qualitative study. BMC Public Health 2010 DOI: 10.1186/1471-2458-10-535.

4. Pew Research Centre. About Seven-in-Ten U.S Adults Say They Need to Take Breaks From COVID-19 News. Available at https://www.pewresearch.org/ journalism/2020/04/29/about-seven-in-ten-u-sadults-say-they-need-to-take-breaks-from-covid-19news/ (accessed January 2021).

5. Agility PR Solutions. Top 10 UK Newspapers by circulation. 2021. Available at https://www.agilitypr. com/resources/top-media-outlets/top-10-uknewspapers-by-circulation/ (accessed January 2021).

6. Gibbons K. Dentists warn of a drastically reduced service. The Times (London) 2020 June 9.

7. Aoraha C. Toothache: Patients face two-year delay to see dentist after waiting times double during coronavirus pandemic. The Sun (London) 2020 October 31.

8. Williams T. Dad downed eight Stellas and pulled his own teeth out as he couldn't see dentist. Metro (London) 2020 October 14.

9. Caulfield C. Fears of spike in 'DIY dentistry' as parents ask how to remove their kids teeth. Metro (London) 2020 April 15.

10. Monti R. Are you willing to try DIY dentistry? We review the at-home remedies to get your teeth stuck into. Daily Mail (London) 2020 September 21.

11. Chalmers V. How a tooth abscess almost killed me: Mother spent five months in hospital after pus inside her mouth led to a life-threatening brain infection. Daily Mail (London) 2020 July 27.

12. Hagan P. Daily Mail. Can dentists survive as the lockdown starts to bite? Shocking report reveals a fifth of practices may close within weeks without a government cash lifeline. Daily Mail (London) 2020 April 13.

13. Abgarian A. What is mask mouth and how can you deal with it? Metro (London) 2020 August 23.

14. Enoch N. Dentists reopen today amid fears patients will face extra fees to pay for $\mathrm{f} 25$ PPE gowns and gloves needed for reach appointment. Daily Mail (London) 2020 June 8.

15. Mills K-A. Mum furious after dentist adds $£ 40$ 'PPE surcharge' to bill for daughter's braces. Mirror (London) 2020 August 6.

16. Phagura S. Outraged patient slams dentist in Bristol after being charged an extra $\mathrm{f} 60$ for treatment to cover PPE COSTS. Daily Mail (London) 2020 September 4.

17. Davis N. 'We should have had time to prepare': dentists on the rush to reopen safely. The Guardian (London) 2020 June 5.

18. Chadwick P. Do media help or harm public health? Aust NZJ Public Health 1998; 22: 155-158.

19. Hooker C, King C, Leask J. Journalists' views about reporting avian influenza and a potential pandemic: a qualitative study. Influenza Other Respir Viruses 2012; 6: 224-229.

20. Medical Defence Union. Doctors subject to criticism in the press. 2014. Available at https://themdu.com/ guidanceandadvice/journals/mdujournalapril-2014/ doctorssubjecttocriticisminthepress (accessed March 2021).

21. Dental Protection. Handling the media: a guide for dentists. Available at https://mpscdnuks.azureedge. net/resources/docs/librariesprovider2/defaultdocument-library/final-2115-dp_uk-handling-themedia_0418_web.pdf (accessed March 2021).

22. Stamm K, Williams Jr J W, Noël P H, Rubin R. Helping journalists get it right: a physicians guide to improving healthcare reporting. J Gen Intern Med 2003; 18: 138-145. 